\title{
Pattern formation by local self-activation and lateral inhibition
}

\author{
Hans Meinhardt* and Alfred Gierer
}

\begin{abstract}
Summary
In 1972, we proposed a theory of biological pattern formation in which concentration maxima of pattern forming substances are generated through local selfenhancement in conjunction with long range inhibition. Since then, much evidence in various developmental systems has confirmed the importance of autocatalytic feedback loops combined with inhibitory interaction. Examples are found in the formation of embryonal organizing regions, in segmentation, in the polarization of individual cells, and in gene activation. By computer simulations, we have shown that the theory accounts for much of the regulatory phenomena observed, including signalling to regenerate removed parts. These selfregulatory features contribute to making development robust and error-tolerant. Furthermore, the resulting pattern is, to a large extent, independent of the details provided by initial conditions and inducing signals.

BioEssays 22:753-760, 2000.

(C) 2000 John Wiley \& Sons, Inc.
\end{abstract}

\section{Introduction}

In 1744, Abraham Trembley, one of the pioneers of developmental biology, discovered that fragments of the freshwater polyp hydra can regenerate complete animals. ${ }^{(1)}$ It turned out that any section cut from the hydra body column regenerates an animal with a head and a foot. These and many subsequent findings on development and regeneration in numerous animal systems raised questions about how striking spatial patterning occurs and how normal development can resume even after severe perturbations such as cutting the organism into pieces. Morphogenetic gradients specifying positional information were postulated, ${ }^{(2)}$ raising again the question of how such gradients are formed in the first place and regulate as they do. Many scientists, up to the time of Spemann in the thirties, had thought that it might be impossible to find an explanation of these phenomena that was based on mechanisms compatible with the known laws of physics and chemistry. Now we know that rather conventional molecular interactions are sufficient. A key contribution was made by Turing who discovered, in 1952,

Max-Planck Institut für Entwicklungsbiologie, Tubingen, Germany. ${ }^{*}$ Correspondence to: Hans Meinhardt, Max-Planck Institut für Entwicklungsbiologie Spemannstr. 35, D-2076 Tübingen, Germany. E-mail: Hans.Meinhardt@tuebingen.mpg.de that the interaction of two substances with different diffusion rates can generate spatial concentration patterns starting from near-uniform initial distributions. ${ }^{(3)}$ He deduced his equation from an analysis of spatial destabilization created by concentration waves of certain wave-lengths. His fundamental discovery initially raised relatively little interest among developmental biologists, perhaps because of the questions raised about the molecular basis and feasibility of the proposed reactions, about the stability of the mathematical solutions, and about the relationship of the solutions to the observed phenomena in developmental regulation. In the early seventies we proposed a theory of biological pattern formation that placed special emphasis on certain striking features of biological development, ${ }^{(4-6)}$ in particular, activating effects (such as induction) and inhibitory effects (such as governing the spacing of structures and substructures). Our equations are of the general reaction-diffusion type introduced by Turing but the essential feature of our approach was the role of autocatalysis in conjunction with lateral inhibition. We have shown that this mechanism accounts for the striking regulation shown by many different organisms including hydra, the experimental model system in our laboratory.

\section{A lesson from vision: amplification of small differences by lateral inhibition}

Our theory was inspired by a concept long discussed in the field of pattern recognition: lateral inhibition. Especially stimulating to us was the work of Reichardt and Kirschfeld in the neighbouring institute of Biological Kybernetics on visual perception in the compound eye of Limulus and insects. The observation that our visual system uses contrast enhancement to detect minute differences in brightness goes back to Ernst Mach in the middle of the last century. ${ }^{(7)} \mathrm{He}$ realized that a change in the slope of a graded brightness is recognized by the observer as a sharp border although this border does not exist in reality. This contrast enhancement is achieved in the retina by a local activation corresponding to the actual stimulus, in conjunction with an inhibitory effect that extends into the surroundings. ${ }^{(8,9)}$ Inside a bright area, the high activation is antagonized by a high inhibition while inside a darker area, the lower activation elicits a lower inhibition. In a region where different intensities are juxtaposed, however, the high activation becomes even higher since the inhibition spreading from the neighbouring darker 
area is low. On the darker side close to the edge, the reversed situation holds: much inhibition spreads from the bright area, causing an even darker appearance. In this way, small differences in contrast become more pronounced. The recognition of the faint contour lines of a series of distant hills, for instance, illustrates the usefulness of this contrast enhancement in human perception.

\section{Long range inhibition on its own is insufficient for pattern formation}

Long range inhibition has long been recognized as an essential element in pattern formation during development. For example, Schoute ${ }^{(10)}$ proposed that the spacing of leaves result from a mutual inhibition of leaf primordia, such that each new leaf can only appear at a certain distance from the preceding one. In the patterning of insect epidermis, Wigglesworth $^{(11)}$ interpreted the spacing of bristles and the insertion of new bristles in the largest interstices in a similar way. Near the turn of the century, by grafting small pieces of hydra tissue, Browne ${ }^{(12)}$ revealed that an existing head has an inhibitory influence on the induction of a second one. This inhibition fades with increasing distance from the existing head. Finally, with the elucidation of the Delta-Notch pathway for the transmission of inhibitory signals, lateral inhibition has become the accepted explanation for the singling out of future sensory bristle cells in Drosophila. ${ }^{(13)}$

Rarely, in the early work, did anyone ask why a leaf, a bristle or a hydra head does not inhibit itself, given that it is in the centre of the inhibition. A remarkable exception is Waddington $^{(14)}$ who rejected the traditional proposal that a hierarchy of self-limiting reactions provides the basis of cellular differentiation ${ }^{(15)}$ because such a scheme neglects the fact that embryonic tissue tends to induce the differentiation of its like rather than to suppress it. He, therefore, proposed the opposite view: "It is in fact more plausible to suggest that differentiation is usually an autocatalytic process" (Ref. 14, p 193).

In processing visual inputs, contrast enhancement works only when a certain pattern is given. The resulting perception must reflect the actual visual input. In contrast, during development the resulting pattern should be, to a large degree, independent of the inducing trigger, i.e. the pattern must be self-generating and self-regulating. Although the absence of any initial asymmetry is a rare situation in normal development, pattern formation is known to occur even under these conditions. One example is the formation of viable polyps from random aggregates of dissociated hydra cells. ${ }^{(16)}$ Therefore, pattern formation must be possible even if no prior pattern is present. In the early sixties, Maruyma ${ }^{(17)}$ emphasized the role of deviation-amplifying processes by positive feedback loops: insignificant or accidental kicks build up and cause significant deviation from the initial conditions. $\mathrm{He}$ interpreted a wide range of processes under this aspect: the development of cities, patterns of erosion, interpersonal processes and international conflicts.

\section{Self-enhancement, a necessary complement of lateral inhibition}

In our adaptation of the neurophysiological concepts to development, we postulated that in addition to long-range inhibition, there is an activation with a strong local selfenhancing component of short range. ${ }^{(4-6)}$ (The range is the mean distance a molecule can travel between its production and disappearance. In the simplest case, it is determined by the diffusion rate and the half-life of the molecule.) In such a system, a homogeneous distribution of the self-enhancing agent is unstable. A minute local increase of the activating substance above the average concentration will grow further due to the self-enhancing process while the concomitantly produced inhibition down-regulates the activation in the surrounding field. A final, stable situation is reached when the local self-enhancement is at equilibrium with the surrounding cloud of inhibition. Even random fluctuations are sufficient to initiate this type of pattern. The simplest molecular realization of this pattern-forming system consists of an autocatalytic activator that also regulates the production of its antagonist: the long-range inhibitor (Fig. 1). Since for any molecular interaction, it is reasonable to assume that the number of molecules disappearing per unit time is proportional to the number of molecules present, the production rate of the activator must contain non-linear elements. This is satisfied, for instance, if the self-enhancing reaction requires a dimerization of the activator, i.e., if two activator molecules have to co-operate to fulfil their function.

Our theory provides a general recipe for designing specific models. It allows for checking hypothetical interactions and their non-linear characteristics as to whether they can lead to stable patterns or not. It also provides an explanation for the paradox mentioned above, namely that a structure can strongly inhibit the same structure in its neighbourhood without inhibiting itself: it is the balance between local non-linear self-enhancement and the dilution of the inhibition, due to its spread into the surroundings that makes the activation resistant to inhibition even at its highest point.

As demonstrated by computer simulations, patterns generated in using our activator-inhibitor system are selfregulating. For instance, if the activated region is removed, the inhibitor-producing region is also removed. After the decay of the remnant inhibitor, a new activation sets in by autocatalysis (Fig. 2). The inhibitor production also resumes, ensuring that the newly emerging activated region assumes the correct profile. Gradients, symmetrical arrangements and periodic distributions can be generated in this way (Fig. 1). The maxima can have a spot- or a stripe-like shape; they can be stable in time, oscillating or they can form travelling

\section{BioEssays 22.8}




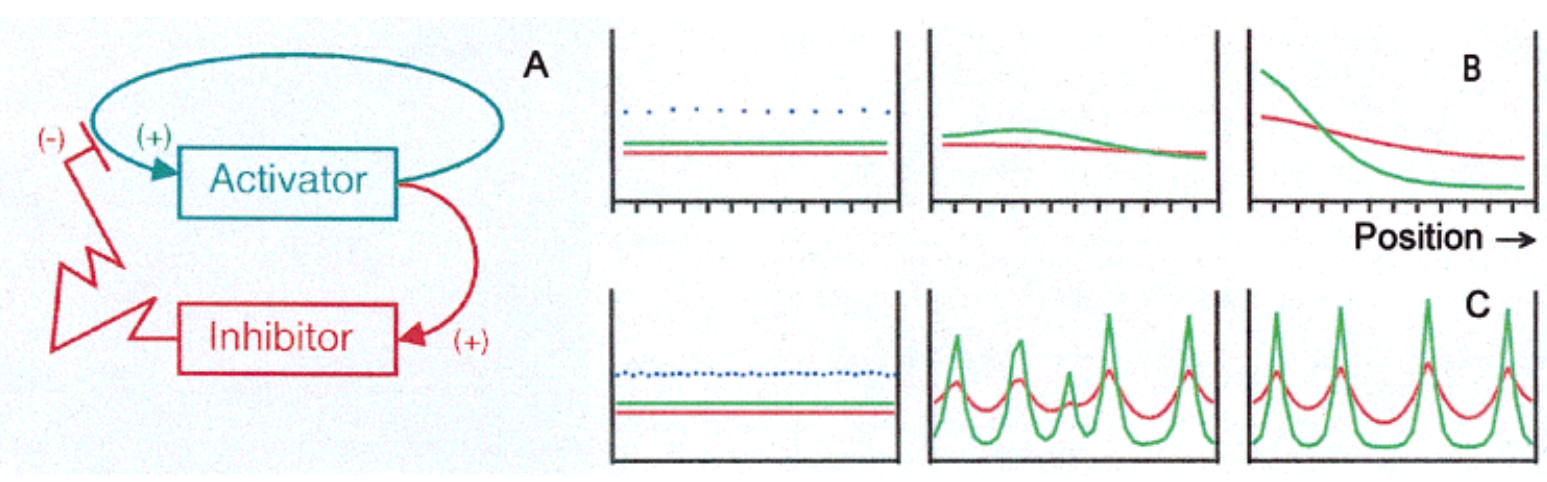

Figure 1. Pattern formation by autocatalysis and long-range inhibition. A: The simplest reaction scheme: an activator (green) catalyses its own production and that of its highly diffusing antagonist, the inhibitor (red). B, C: Computer simulation of pattern formation in a chain of cells. Random fluctuation in the ability of the cells to perform the reaction (blue squares) are sufficient to initiate pattern formation. The initial, an intermediate and the finally stable distributions are shown. B: In a field of the size not much above the activator range, only a graded distribution is possible. C: In a field larger than the range of the inhibitor, several maxima emerge. Under this condition, the spacing is somewhat irregular but a maximum and minimum distance is maintained (details and software for simulations are given in Ref. 18).

waves. These latter modes have been used to explain the generation of the beautiful pigmentation patterns on tropical sea shells. ${ }^{(18)}$ Further, there are several ways within the theory to endow a pattern-forming system with the capability of proportion regulation. In such a case, the extension of an activated region is always a certain fraction of the field size.

From the beginning, our activator-inhibitor approach was directly linked to biological developmental regulation. In contrast, Turing's approach was essentially mathematical. He used a Fourier-type stability analysis of linear equations based on the amplification of waves with certain spatial wave-lengths after small perturbations. Although his theory was not originally based on autocatalysis and lateral inhibition, in an unpublished note found after his death, there are sentences alluding to inhibitory principles: The amplitude of waves is largely controlled by the concentration $V$ of 'poison'.(19) On the other hand, we did not exploit the potential of Fourier wave analysis in our original papers. The relation between the two approaches is by no means obvious. Only a close analysis has shown that the mathematical content of both lines of thought is very similar. $^{(5,20,21)}$ The equation with which Turing exemplified his theory can also satisfy the condition of self-activation with lateral inhibition. In retrospect, we may consider the latter principle as a specification of conditions for pattern formation based on reaction-diffusion mechanisms. From a different perspective, however, one can regard molecular reactiondiffusion mechanisms as a special case of the more general principle underlying the generation and maintenance of many structures. It is applicable not only to developmental processes but also to patterns of neural activity, spatiotem- poral population dynamics, ${ }^{(22)}$ formation of sand dunes and other inorganic patterning, ${ }^{(23)}$ the rise of towns and also of various psychological processes.

Our lateral inhibition rules subsume indirect inhibition resulting from a depletion of a substance required for the autocatalytic process. ${ }^{(24)}$ With this one generalization, for the simplest two-factor case, our rules can be shown mathematically to be the only mode of pattern generation.

What about pattern formation in systems with more than two variables? Biologically, this is the most likely situation. Feedback loops can consist of long chains of reactions. What is our criterion for pattern formation in this situation? We might think of collecting those compounds that have autocatalytic effects and analysing them individually but this approach leads nowhere. For instance, activation can result just from two substances that inhibit each other mutually, allowing for pattern formation even if there is not a single directly self-enhancing reaction. A more adequate approach is different: apply the lateral inhibition concept from the outset and begin the analysis by sorting the molecules involved into those with a short range on the one hand, and those subject to a wider distribution in the tissue on the other. Pattern formation occurs if the short-range subset, taken together, is in itself autocatalytic as a system whereas the system as a whole, including the long-range components, must be able to prevent an overall autocatalytic explosion. In this way, the concept of "pattern formation by the conjunction of activation and inhibition" can be generalized into multi-component systems, with activation and inhibition as features of subsystems rather than of individual substances. ${ }^{(5)}$ Nevertheless, the basic regula- 


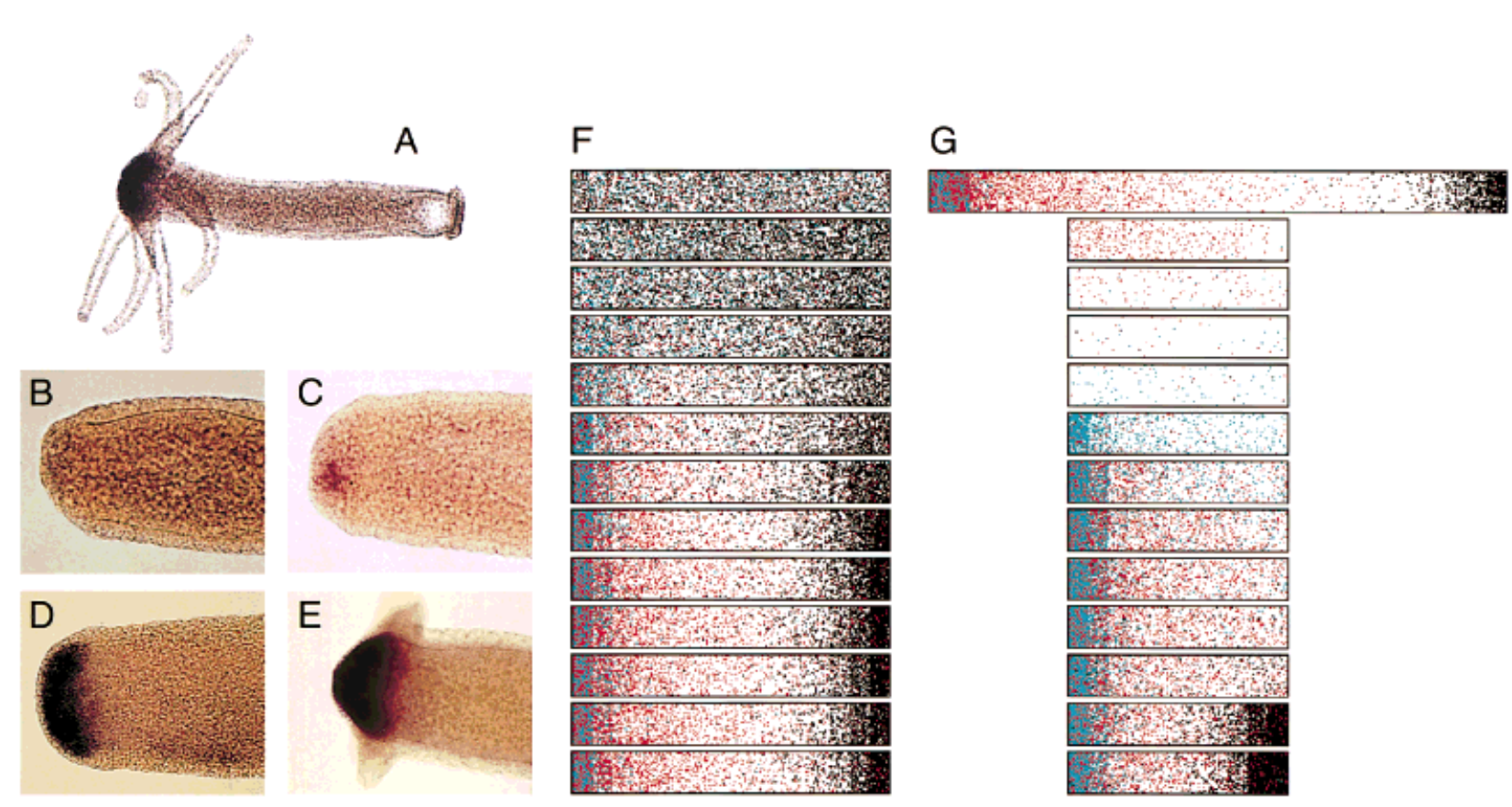

Figure 2. Generation and regeneration of the head signal in hydra. A: HyBra1 is indicative for a head forming signal. ${ }^{(45)}$ B-E: After head removal, the signal reappears after $3 \mathrm{~h}(\mathrm{C})$, it is fully present at $4 \mathrm{~h}(\mathrm{D})$ and after $48 \mathrm{~h}$, tentacles become visible (E). F,G: Computer simulation of a pattern formation from a near homogeneous initial situation and regeneration. The density of dots indicates local concentrations. Shown are successive time steps. A high concentration of the autocatalytic head activator (blue) emerges on one side of the field. It is in an dynamic equilibrium with the long ranging inhibitor (red). By an appropriate coupling the foot activator (black) appears at the opposite end of the field. ${ }^{(58)}$ After head removal $(\mathrm{G})$, first the remnant inhibitor decays. Due to autocatalysis, a new activation is "fired" that become shaped by the concomitantly produced inhibitor. Somewhat delayed, the foot activator regenerates too. Animated simulations are available at http://www.eb.tuebingen.mpg.de/abt.4/meinhardt/theory.html. (Photographs A-E kindly supplied by U. Technau).

tory capabilities that characterize biological development are maintained. In the course of time, a substantial body of literature has built up around pattern forming reactions. ${ }^{(25-27)}$

\section{Pattern formation within cells and tissues are based on the same principles}

Pattern formation takes place not only in fields of cells but also within single cells. The mechanism described above is appropriate for intracellular activation if the spread of the self-enhancing component is restricted within the cell, for instance, by an attachment to the cell cortex. In contrast, the antagonistic reaction has to spread more freely, perhaps within the cytoplasm. The egg of the brown algae Fucus is a well-investigated model system for pattern formation in single cells. The cell is initially almost symmetric. The localization of the outgrowth of the rhizoid and the orientation of the first cell division require a pattern-forming event. Almost any external cue can orient this pattern ${ }^{(28)}$ but the resulting pattern is independent of the trigger. In the absence of an external asymmetry, the polarization occurs in a somewhat delayed fashion and with a random orientation, suggesting that the symmetric situation is unstable and a pattern has to be formed. The first reliable indications of the polarization of the cell are localized currents of $\mathrm{Ca}^{2+}$ ions. ${ }^{(29)}$ Artificial asymmetric permeabilization by application of calcium ionophores can orient the outgrowth of the Fucus egg $^{(30)}$ and that of growth cones. ${ }^{(31)}$ Currents of $\mathrm{Ca}^{2+}$ ions are well known to have self-enhancing components, ${ }^{(32)}$ suggesting a link to the proposed mechanism for pattern formation. In a somewhat more complex form, the theory can describe the maintenance of the high sensitivity against external cues that is characteristic of and required for chemotactic cells or navigating growth cones. ${ }^{(33)}$

\section{Molecular candidates for activator-inhibitor systems}

With the advances in gene cloning and the isolation of relevant molecules, several systems have emerged that are good candidates for activator-inhibitor systems. In this section we present examples of such systems. The following

\section{BioEssays 22.8}


list is certainly not exhaustive and the function assigned to one or the other component may change in the future. The list is intended to demonstrate only that a strong correspondence has emerged between the molecules found and the ingredients predicted by our theory.

A principal element in the formation of founder cells of the regularly arranged ommatidia of the Drosophila eye is the gene atonal. This gene contains an autoregulatory element based presumably on a direct binding of the Atonal protein to the enhancer region. ${ }^{(34)}$ The antagonistic reaction in this example depends on the Delta-Notch system, scabrous ${ }^{(35)}$ and hedgehog. ${ }^{(36)}$ Similarly, the key gene for the formation of sensory bristles in Drosophila, the achaete-scute complex, contains enhancer elements that bind the Scute protein. ${ }^{(37)}$ In this system the inhibition also works via the Delta-Notch system. ${ }^{(13)}$ Recently, it has been shown that Delta is not only a ligand expressed on the cell surface but that it can be cleaved off and spread over several cell diameters. ${ }^{(38)}$ Similar reactions have been found in the spacing of hairs on the leaves of Arabidopsis. ${ }^{(39)}$

In the blue-green algae Anabaena, about every seventh cell differentiates to a nitrogen-fixating cell termed heterocyst. Whenever two heterocyst cells become separated (due to cell proliferation) by more than about ten cells, an interstitial cell also differentiates into a heterocyst. ${ }^{(40,41)} \mathrm{We}$ now know that there is a small protein that acts as an inhibitor of this process. Over-expression of this protein leads to a general down-regulation of heterocyst formation, while abolishing its action causes almost all cells to differentiate into heterocysts. ${ }^{(42)}$ These results are in full agreement with the expectations from the model.

Similar interactions can also be found in higher organisms. In the generation of highly regular feather patterns, BMP-2 and BMP-4 are involved in the lateral inhibition, ${ }^{(43)}$ while FGF-4 and sonic hedgehog are elements of the activating mechanism. ${ }^{(44)}$ In these investigations, the regions in which genes for activating and inhibiting molecules are transcribed were determined. As expected from the theory, these regions coincide in most cases. A more direct comparison with the theory, however, would require knowledge of the actual ranges of the signalling molecules involved.

Of particular interest are molecules likely to be involved in the primary pattern formation of hydra. As shown by Technau and Bode ${ }^{(45)}$ the brachyury homologue HyBra1 is transcribed in the head region. Upon head removal, the activity of this gene reappears after 3-4 h (Fig. 2). In vertebrates an autocatalytic loop exists between the transcription factor brachyury and the secreted factor FGF, a reaction enabling a spread of the self-enhancing reaction. ${ }^{(46)}$ Whether a similar loop exists in hydra is not yet known. While the inhibitory molecules identified so far in other systems are only of short range, experimental evidence suggests that in this case the range can be quite large. An existing head of a hydra inhibits head formation along the entire body column. The molecular basis of such long-range inhibitory signalling is still unknown.

An example of pattern formation by activation resulting from inhibition of inhibition may be the patterning system that leads to the separation of the axial and more lateral structures in vertebrate development. The key components of two pathways, chordin/noggin/follistatin on the one hand and BMP-4 on the other, inhibit each other mutually. This mutual inhibition may represent, in fact, a hidden selfactivation. Such reactions by themselves could only work as a switch in which either one or the other component wins. To ensure that both regions are formed in the correct proportions, a long-range antagonistic interaction is again required. A BMP-3 related molecule has been found that has the corresponding anti-dorsalizing action. ${ }^{(47,48)}$ This finding has been regarded as a paradox: molecules are produced in the organizing region that suppress organizer formation when over-expressed. This is exactly what is expected, however, if in the wild-type situation, self-enhancement and inhibition balance each other. Again, although the antagonist has to spread further, the region in which the activator and the antagonist are produced should be identical.

\section{Pattern formation and cell response: related mechanisms cause reliability}

The activator-inhibitor mechanism described above allows the generation of graded concentration profiles that can instruct cells about their positions in a field. In other words, it allows the generation of positional information. Remarkably, the response of the cells (i.e. the activation of genes to achieve a position-specific cell differentiation) requires the same components: autoregulation and competition. In a particular developmental situation, a group of cells may have different options for its fate. These options become realized by activation of particular genes. To make the correct decisions under the influence of a morphogenetic gradient, minute concentration differences must be sufficient for the difference in gene expression. The formal correspondence between pattern formation and gene activation is easy to see. Patterning in space requires activation at a particular location and inhibition in the remaining field. Analogously, the selection of a particular pathway requires the activation of the gene required for the particular pathway and the suppression of those genes that would lead to all other pathways available to the cell in this situation. Based on this analogy, it has been proposed that stable gene activation requires autoregulation of genes as well as the competition among alternative genes. ${ }^{(4)}$ Since then, many genes have been found that are regulated in this way. The genes Deformed ${ }^{(50)}$ or twist ${ }^{(51)}$ are examples. Such an interaction leads to an unambiguous response by the cells with well-defined thresholds, and allows the maintenance of gene activation even 
when the evoking signals are no longer present. These two features contribute substantially to the reliability of development.

\section{Segmentation: lateral inhibition by a long-range promotion of an antagonistic feedback loop}

The final stage of segmentation in insects can be described in terms of a mutual activation of cell states that locally exclude each other. The self-enhancing feature in such multicomponent systems is the difference of activation between neighboring areas. Our model ${ }^{(52)}$ has found support by the elucidation of the engrailed/wingless interaction. ${ }^{(53)}$ Lateral inhibition works in this case not by a direct suppression of the same autocatalytic loop in the neighbourhood but by the lateral activation of a second autocatalytic loop that, in turn, locally suppresses the first. This leads to a situation in which two adjacent cell states need each other in a symbiotic manner. If one of the loops is defective due to a mutation, the other degenerates also, in full agreement with the observation.

\section{The role of initial asymmetries}

Although not required for de novo pattern formation, initial asymmetries are found in many biological systems. Hydra regeneration provides a good example. Fragments of the body column regenerate complete organisms with head and foot. The orientation of the pattern is determined by the polarity of the regenerating tissue: the head appears always on the side pointing originally towards the head. In other words, it is the relative position of a cell within the fragment that determines whether it will participate in the formation of a head, a foot, or whether it will remain a part of the body column. We attribute this polarity to a gradient in the ability of the tissue to perform the pattern-forming reaction, a property we have called source density. The source density gradient extends across the tissue from head to foot. Hence, the apical part of a fragment always has an advantage in the competition to regenerate the head activation and this part will always win. The initial asymmetry only orients the pattern. In contrast, the profile of the head-activation signal is self-regulating and independent of the asymmetry. In the course of regeneration, the head activation reappears first. Under its influence, the source gradient becomes reestablished as a slow response. This process prevents the asymmetry within the tissue from being diluted out during several rounds of regeneration. As one can see, the theory describes not only how the head-inducing signal can be formed and maintained in a dynamic way but also how the tissue can maintain its polarity.

A source gradient within a tissue may be of biological significance in several ways. For the development of a polar structure, no time-consuming competition is required. Due to the pronounced advantage of one side, it is clear which site will become activated. Further, a single organizing region can be maintained even if the total extension of the field increases due to growth beyond the mean-range of the inhibitor. Regions further away from the organizing region become less and less competent to perform the autocatalysis, rendering a spontaneous formation of a secondary organizing region less likely. So far, in hydra we do not know the molecular basis of this asymmetry but molecules are known that interfere with this graded property. After treatment with Diacylglycerol, for instance, the whole tissue obtains a near-head character. ${ }^{(54)}$ Multiple heads and isolated tentacles are formed, demonstrating that the apical dominance has been lost. This observation suggest that the source density is sensitive to manipulation of the second messenger pathway.

Is self-organization by autocatalysis and lateral inhibition also involved in Spemann's organizer? In contrast to comparatively simple systems of de novo pattern formation such as regenerating hydra tissue, organizer formation and function are complex processes. Yet, it appears that selforganization of patterns plays a crucial role, though the competence for such processes is restricted in space and time during development. The Spemann organizer in amphibians is an example. An early removal of organizer can be compensated ${ }^{(55)}$ but only as long as the removed sector is smaller than $30^{\circ}$. Nevertheless, the work by Nieuwkoop ${ }^{(56)}$ convincingly demonstrated that the amphibian organizer is based on a pattern-forming reaction. He made aggregates from dissociated animal and vegetal cells. Their juxtaposition lead not only to the induction of mesodermal tissue along the common border but also to the formation of one or more new organizing regions within this new mesodermal zone. Therefore, organizing regions can be formed even after any spatial cues imposed by sperm entry and cortical rotation are eliminated (by dissociation and reaggregation). Organizer formation does not require prelocalized determinants but can also happen when cells that are capable of generating the pattern-forming reaction become randomly distributed. What is important is that the prerequisites are present, not that they are spatially localized.

Further support for the view that organizer formation is based on a genuine patterning process comes from the chicken and mouse developments. In early chick development, a separation of the blastodisc into several fragments leads to the formation of separate embryos in each fragment, ${ }^{(57)}$ a regulation analogous to the regeneration in hydra. Occasionally, two embryos are also formed spontaneously. They appear at opposite positions, so that one of the embryos appears at a side disfavoured by the initial asymmetry imposed by gravity. This, however, is expected if long-range inhibition plays an important role. In the mouse, there is little initial asymmetry in the fertilized egg and a

\section{BioEssays 22.8}


spontaneous splitting of the early embryo can lead to identical twins, indicating that the final stage of mouse development is not under the control of a fixed deposition of determinants.

\section{Conclusions}

Development requires a chain of hierarchically nested reactions, each depending on previous steps and controlling subsequent steps. The general scheme of local selfenhancement and long-range inhibition seems to be a very important component in making this chain reliable and errortolerant. Growing evidence shows the recurrence of this general scheme in many different developmental situations, from single cells to mammalian embryology. We hope that our concepts will facilitate the understanding of the many complex-appearing webs of molecular interactions involved in development.

\section{References}

1. Trembley A. Memoires pour servir a l'histoire d'un genre de polypes d'eau douce. J. u. H. Verbeek, Leiden 1744.

2. Wolpert L. Positional information and the spatial pattern of cellular differentiation. J Theor Biol 1969;25:1-47

3. Turing A. The chemical basis of morphogenesis. Phil Trans B 1952; 237:37-72

4. Gierer A, Meinhardt H. A theory of biological pattern formation Kybernetik 1972;12:30-39.

5. Gierer A. Generation of biological patterns and form: Some physical, mathematical and logical aspects. Prog Biophys Molec Biol 1981;37: $1-47$.

6. Meinhardt H. Models of biological pattern formation, Academic Press, London, 1982

7. Mach E. Über die Verteilung des Lichtreizes auf die Netzhaut. Sitzungsberichte Math Naturwiss KI Wien 1865;52:303-322.

8. Hartline HK, Wagner HG, Ratliff F. Inhibition in the eye of limulus. J Gen Physiol 1956;39:651-673

9. Kirschfeld B, Reichardt W. Die Verarbeitung stationärer optischer Nachrichten im Komplexauge von Limulus. Kybernetik 1964;2: 43-61.

10. Schoute JC. Beiträge zur Blattstellung. Rec Trav Bot Neerl 1913;10: 153-325.

11. Wigglesworth VB. Local and general factors in the development of "pattern" in Rhodnius prolixus. J Exp Biol 1940;17:180-200.

12. Browne EN. The production of new hydrants in Hydra by insertion of small grafts. J Exp Zool 1909;7:1-23.

13. Simpson P. Notch signalling in development-on equivalence groups and asymmetric developmental potential. Curr Opin Genet Dev 1997;7: $537-542$.

14. Waddington $\mathrm{CH}$. Principles of Embryology. London. Georg Allen and Unwin Ltd, 1996.

15. Rose SM. A hierarchy of self-limiting reactions as the basis of cellular differentiation and growth control. Amer Nat 1952;86:337.

16. Gierer A, Berking S, Bode H, David CN, Flick K, Hansmann G, Schaller $\mathrm{H}$, Trenkner E. Regeneration of hydra from re-aggregated cells. Nat New Biol 1940;239:98-101.

17. Maruyama M. The second cybernetics: deviation-amplifying mutual causal processes. Amer Sci 1963;51:164-179.

18. Meinhardt H. The Algorithmic Beauty of Sea Shells. Heidelberg New York. Springer 1998 (with PC-software)

19. Hodges A. Alan Turing: the enigma, Simon and Schuster. New York, 1983 (quotation on page 494).

20. Babloyanz A, Hiernaux J. Models for cell differentiation and generation of polarity in diffusion governed morphogenetic fields. Bull Math Biol 1975;7:637-657.
21. Granero MI, Porati A, Zanacca D. A bifurcation analysis of pattern formation in a diffusion governed morphogenetic field. J Math Biol 1977; 4:21-27.

22. Segel LA, Jackson JL. Dissipative structure: an explanation and an ecological example. J Theor Biol 1972;37:545-549.

23. Ball P.The self-made tapestry-pattern formation in nature. Oxford, New York, Tokyo. Oxford University Press. 1999

24. Prigogine I, Lefever R. Symmetry breaking instabilities in dissipative systems. II J Chem Phys 1968;48:1695-1700.

25. Fife PC. Mathematical Aspects of Reacting and Diffusing Systems. Lecture Notes in Biomathematics. Berlin, Heidelberg, New York. Springer-Verlag. 1979.

26. Murray JD. Mathematical Biology. Heidelberg, New York. Springer. 1989.

27. Harrison LG. Kinetic theory of living pattern. Cambridge. Cambridge Univerity Press. 1993.

28. Goodner B, Quatrano RS. Fucus embryogenesis - a model to study the establishment of polarity. Plant Cell 1993;5:1471-1481.

29. Jaffe LF. Organization of early development by calcium patterns. Bioessays 1999;21:657-667.

30. Robinson KR, Cone R. Polarization of Fucoid eggs by a calcium ionophore gradient. Science 1980;207:77-78.

31. Anglister L, Farber IC, Shahar A Grinvald A. Localization of voltagesensitve Calcium channels along developing neurites: their possible role in regulating neurite elongation. Dev Biol 1982;94:351-365

32. Berridge MJ. Inositol triphosphate and calcium signalling. Nature 1993; 361:315-325

33. Meinhardt $\mathrm{H}$. Orientation of chemotactic cells and growth cones: Models and mechanisms. J Cell Sci 1999;112:2867-2874.

34. Sun Y, Jan LY, Jan YN. Transcriptional regulation of atonal during development of Drosophila peripheral nervous system. Development 1998; 125:3731-3740

35. Lee EC, Hu XX, Yu SY, Baker NE. The scabrous gene encodes a secreted glycoprotein dimer and regulates proneural development in Drosophila eyes. Molec Cell Biol 1996;16:1179-1188.

36. Dominguez M. Dual role for Hedgehog in the regulation of the proneural gene atonal during ommatidia development. Development 1999;126: 2345-2353.

37. Culi J, Modolell J. Proneural gene self-stimulation in neural precursorsan essential mechanism for sense organ development that is regulated by Notch signalling. Genes Dev 1998;12:2036-2047.

38. Qi HL, Rand MD, Wu XH, Sestan N, Wang WY, Rakic P, Xu T, ArtavanisTsakonas S. Processing of the Notch ligand Delta by the metalloprotease kuzbanian. Science 1998;283:91-94.

39. Schnittger A, Folkers U, Schwab B, Jürgens G, Hülskamp M. Generation of spacing pattern: The role of Triptychon in trichome patterning of Arabidopsis. Plant Cell 1999;11:1105-1116.

40. Wilcox M, Mitchison GJ, Smith RJ. Pattern formation in the blue-green alga Anabaena, I, Basic mechanisms. J Cell Sci 1998;12:707-723.

41. Wolk CP, Quine MP. Formation of one-dimensional patterns by stochastic processes and by filamentous blue-green algeae. Dev Biol 1975;46:370-382.

42. Yoon HS, Golden JW. Heterocyst pattern-formation controlled by a diffusible peptide, Science 1975;282:935-938.

43. Noramly S, Morgan BA. BMPs mediate lateral inhibition at successive stages in feather tract development. Development 1998;125: 3775-3787

44. Jung HS, Franciswest PH, Widelitz RB, Jiang TX, Tingberreth S, Tickle $C$, Wolpert L, Chuong CM. Local inhibitory-action of BMPs and their relationships with activators in feather formation-implications for periodic patterning. Dev Biol 1998;196:1-23.

45. Technau U, Bode HR. HyBra1 a Brachyury homologue acts during head formation in Hydra. Development 1999;126:999-1010.

46. Schulte-Merker S, Smith JC. Mesoderm formation in response to brachyury requires FGF signaling. Curr Biol 1995;5:62-67.

47. Moos M, Wang SW, Krinks M. Anti-dorsalizing morphogenetic protein is a novel TGF-beta homolog expressed in the Spemann organizer. Development 1995;121:4293-4301.

48. Joubin K, Stern CD. Molecular interactions continuously define the organizer during the cell movements of gastrulation. Cell 1999;98: $559-571$ 
49. Meinhardt $H$. Space-dependent cell determination under the control of a morphogen gradient. J Theor Biol 1978;74:307-321.

50. Regulski M, Dessain S, McGinnis N, McGinnis W. High-affinity bindingsites for the deformed protein are required for the function of an autoregulatory enhancer of the deformed gene. Genes Dev 1991;5: $278-286$.

51. Leptin M. twist and snail as positive and negative regulators during Drosophila mesoderm development. Genes Dev 1991;5: 1568-1576.

52. Meinhardt $H$, Gierer A. Generation and regeneration of sequences of structures during morphogenesis. J Theor Biol 1980;85:429450 .

53. Ingham PW, Martinez-Arias A. Boundary and fields in early embryos. Cell 1992;68:221-235
54. Müller WA. Diacylglycerol-induced multihead formation in hydra. Development 1989;105:309-316.

55. Steward RM, Gerhart JC. The anterior extent of dorsal development of the Xenopus embryonic axis depends on the quantity of organizer in the late blastula. Development 1990;109:363-373.

56. Nieuwkoop PD. The formation of the mesoderm in urodelean amphibians, VI, The self-organizing capacity of the induced meso-endoderm, Roux's Arch. Dev Biol 1992;201:18-29.

57. Lutz $\mathrm{H}$. Sur la production experimentale de la polyembryonie et de la monstruosite double ches lez oiseaux. Arch d'Anat Micro et de Morph 1949;38:79-144

58. Meinhardt $\mathrm{H}$. A model for pattern-formation of hypostome tentacles and foot in hydra: how to form structures close to each other how to form them at a distance. Dev Biol 1993;157:321-333.

\section{BioEssays 22.8}

